OPEN ACCESS

Edited by:

Ce Dou,

Army Medical University, China

Reviewed by:

Jiacan Su,

Second Military Medical University,

China

Yinhe Wang

Nanjing University Medical School,

China

${ }^{*}$ Correspondence:

Peng Xue

hebmuxuepeng@163.com

Sijing Liu

hbydxb1@126.com

${ }^{t}$ These authors have contributed equally to this work and share first

authorship.

Specialty section:

This article was submitted to

Cellular Biochemistry,

a section of the journal

Frontiers in Cell and Developmental

Biology

Received: 30 August 2021 Accepted: 05 November 2021 Published: 03 December 2021

Citation:

Wang N, Li Z, Li S, Li Y, Gao L, Bao X, Wang K, Liu C, Xue P and Liu S (2021) Curculigoside Ameliorates Bone Loss by Influencing Mesenchymal Stem Cell

Fate in Aging Mice.

Front. Cell Dev. Biol. 9:767006.

doi: 10.3389/fcell.2021.767006

\section{Curculigoside Ameliorates Bone Loss by Influencing Mesenchymal Stem Cell Fate in Aging Mice}

\author{
Na Wang ${ }^{1,2 \dagger}$, Ziyi $\mathrm{Li}^{1,2+}$, Shilun $\mathrm{Li}^{3 \dagger}$, Yukun $\mathrm{Li}^{1,2}$, Liu Gao ${ }^{1,2}$, Xiaoxue Bao ${ }^{1,2}, \mathrm{Ke}$ Wang ${ }^{1,2}$, \\ Chang Liu ${ }^{1,2}$, Peng Xue ${ }^{1,2 *}$ and Sijing Liu ${ }^{4 *}$ \\ ${ }^{1}$ Department of Endocrinology, The Third Hospital of Hebei Medical University, Shijiazhuang, China, ${ }^{2}$ Key Orthopaedic \\ Biomechanics Laboratory of Hebei Province, Shijiazhuang, China, ${ }^{3}$ Department of Joint Surgery, The Third Hospital of Hebei \\ Medical University, Shijiazhuang, China, ${ }^{4}$ Editorial Department of Hebei Medical University, Hebei Medical University, \\ Shijiazhuang, China
}

Senile osteoporosis is characterized by increased bone loss and fat accumulation in marrow. Curculigoside (CCG) is the major bioactive component of Curculigo orchioides, which has been used as anti-osteoporosis therapy for elder patients since antiquity. We aimed to investigate the underlying mechanisms by which CCG regulated the bone-fat balance in marrow of aging mice. In our study, CCG treatment was identified to interfere with the stem cell lineage commitment both in vivo and in vitro. In vivo, CCG promoted the transcriptional co-activator with PDZ-binding motif (TAZ) expression to reverse age-related bone loss and marrow adiposity. In vitro, proper concentration of CCG upregulated TAZ expression to increase osteogenesis and decrease adipogenesis of bone marrow mesenchymal stem cells (BMSCs). This regulating effect was discounted by TAZ knockdown or the use of MEK-ERK pathway inhibitor, UO126. Above all, our study confirmed the rescuing effects of CCG on the differential shift from adipogenesis to osteogenesis of BMSCs in aging mice and provided a scientific basis for the clinical use of CCG in senile osteoporosis.

Keywords: osteoporosis, curculigoside, BMSCs, TAZ, MEK-ERK pathway

\section{INTRODUCTION}

Osteoporosis is increasingly recognized as a major health concern that affects approximately $50 \%$ of women and 20\% of men over 50 years old (Sambrook and Cooper, 2006). This age-related systemic impairment of bone loss is characterized by decreased osteogenesis and increased adiposity in bone marrow, resulting in more propensity of fragility fracture (Hu et al., 2018; Sanghani-Kerai et al., 2018; Zanker and Duque, 2019). Bone marrow mesenchymal stem cells (BMSCs) could differentiate to osteoblasts or adipocytes. They held a competition relationship during BMSCs' differentiation (Ambrosi et al., 2017; Muruganandan et al., 2017). The process of stem cell fate determination is constantly changing, and consists of self-renewal and terminal differentiation (Hansen et al., 1999; Dalton, 2015; Urbach and Witte, 2019). Various exogenous and endogenous factors could alter the differentiation switch from adipogenesis to osteogenesis of BMSCs context-dependently (Teitelbaum, 2010; Huang et al., 2018; Wang et al., 2019a). However, the underlying mechanism has not been clearly addressed.

Curculigo orchioides, a small herbal plant belonging to the family Amaryllidacea, has been used as an anti-osteoporosis herb in many Asian countries (Wang et al., 2012; Wu et al., 2012; Tan et al., 2019). The 
extracts of this plant contain a wide variety of flavonoids, phytosterols, and phenolic compounds, with curculigoside (CCG) identified as the main active substance (Wang et al., 2017). In vivo study, CCG was reported to distribute widely in bone marrow and many other tissues after oral administration (Yuan et al., 2015). Cao et al. (2008) discovered that oral administration of CCG prevented bone loss in the tibia of the ovariectomized rats. As was demonstrated, CCG could up-regulate VEGF expression, reverse iron-overload via GPX4, relieve oxidative stress via Akt-FoxO1 axis, and reduce inflammation via NF- $\kappa \beta$ signaling transduction to favor the bone remodeling in vivo and in vitro (Cao et al., 2008; Ma et al., 2011; Tan et al., 2019; Zhang et al., 2019; Wang et al., 2020). In our research, we tried to elucidate if and how CCG switch the differentiation from adipogenesis to osteogenesis of BMSCs in aging mice.

Senile osteoporotic patients often showed an increased adipogenesis in their bone marrow with a reduction of osteoblastogenesis (Rosen et al., 2009). Transcriptional coactivator with PDZ-binding motif (TAZ) has been demonstrated as a nuclear transcription factor playing crucial roles in stem cell differentiation (Kegelman et al., 2018; Wang et al., 2018; Tan and Dai, 2019). As was proved, TAZ combined to runt-related transcription factor 2 (RUNX2) to promote osteogenesis, and interacted with peroxisome proliferator-activated receptor $\gamma$ (PPAR $\gamma$ ) to suppress adipogenesis (Byun et al., 2013; Matsumoto et al., 2016; Wang et al., 2019b). Besides, TAZ has neuro-protection, angiogenesis, and anti-oxidative stress effects, which were important for bone nutrition and regeneration (Lee et al., 2019; Chen et al., 2021; Jeanette et al., 2021). Yu et al. (2018) revealed that PGC-1 alpha targeted TAZ to alter bone-fat balance during skeletal aging. Previously, our study also pointed a molecular link of MEK-ERK pathway to the TAZ during BMSCs osteogenesis (Wang et al., 2018; Wang et al., 2019a). As the MEK-ERK signaling pathway has been intensively investigated in regulating cells differentiation, it exerts our interest on the role of MEK-ERK/TAZ axis in the CCGmediated differentiation of BMSCs during aging.

Molecular interactions and protein networks of CCG could predict the functions of its compounds as an anti-osteoporotic drug in clinical. However, the underlying mechanisms whereby CCG promotes osteogenesis in bone marrow have not been well established. In this study, we found that CCG induced the switch from adipogenesis to osteogenesis of BMSCs in aging mice and ameliorated the age-related bone loss by influencing the osteoblastsadipocytes lineage commitment of BMSCs via MEK/ERK-TAZ interaction axis. Altogether, these findings provided a scientific basis for the clinical use of CCG in the geriatric population.

\section{MATERIALS AND METHODS}

\section{Mice and In Vivo Treatment}

Our experiments were approved by the Local Committee of Animal Use and Protection of the Third Hospital of Hebei Medical University. C57BL/6 mice were bred in a room with the environmental temperature ranging from 20 to $24^{\circ} \mathrm{C}$ and proper humidity ranging from 30 to $70 \%$. The mice had either free access to food or water. The 3-months mice were regarded as the normal control group. In addition, the three groups of 16months mice would be received vehicle, $50 \mathrm{mg} / \mathrm{kg} / \mathrm{d}$ or $100 \mathrm{mg} / \mathrm{kg} / \mathrm{d}$ CCG (Shanghai Standard Biotech, Shanghai, China) by oral administration, respectively, for 2 months. After the 2 months treatment, we considered the above three groups as 18 -mon, $50 \mathrm{mg}$-CCG, and $100 \mathrm{mg}$-CCG group.

\section{Micro-Computed Tomography $(\mu \mathrm{CT})$ Analyses}

We fixed the harvested femurs in $4 \%$ paraformaldehyde $\left(4^{\circ} \mathrm{C}, 24 \mathrm{~h}\right)$. We used the SkyScan $\mu \mathrm{CT}$ scanner to analyze the bone parameters with $65 \mathrm{kv}$ voltage and $153 \mu \mathrm{A}$ current. Each image was obtained with high resolution of $9.0 \mu \mathrm{m}$ per pixel. We used NRecon for image reconstruction (version 1.6), CTAn (version 1.9) for data analysis, and CTVol (version 2.0) for 3D model visualization. With 3D analysis, we collected bone parameters of trabecular bone volume fraction (BV/TV), trabecular bone thickness (Tb.Th), trabecular bone number (Tb.N), and trabecular bone separation (Tb.Sp) to represent the femur character in this part.

\section{OsO4 Staining and $\mu \mathrm{CT}$ Analysis}

We decalcified the long bone in 0.5 M EDTA for 21 days. We cut off the proximal of femurs and then discarded it. The rest of the femur was incubated in aqueous osmium tetroxide (OsO4) (2\%, $2 \mathrm{~h}$ ). Then we rinsed the tissues for $48 \mathrm{~h}$ and scanned them by using $\mu \mathrm{CT}(9-\mu \mathrm{m}$ pixel; $45 \mathrm{kV}, 177 \mu \mathrm{A})$. The number and the volume of adipocytes ( $\mathrm{ad} . \mathrm{N}$; ad. $\mathrm{V}$ ) in bone marrow were quantified for inter-group analyses in this part.

\section{Hematoxylin and Eosin and Tartrated Resistant Acid Phosphatase Staining}

After 3-weeks decalcification of the femur samples, we embedded them in paraffin and cut them into $4 \mu \mathrm{m}$-thick slices for $\mathrm{H} \& \mathrm{E}$ and TRAP staining. The adipocyte number relative to bone marrow area was then collected for inter-group analysis based on the $\mathrm{H} \& \mathrm{E}$ staining; and the osteoclast (OC) numbers were calculated via TRAP staining.

\section{Immunofluorescence Assay}

Harvested femurs were decalcified by $0.5 \mathrm{M}$ EDTA. The tissues were embedded in gelatin (at a concentration of $8 \%$ ) with sucrose (at a concentration of 20\%) in the presence of polyvinylpyrrolidone (at a concentration of $2 \%$ ). We sliced the tissues into $20 \mu \mathrm{m}$-thick sections. Subsequently, we blocked the samples, treated the samples with primary antibodies (the antibodoy for TAZ, 1:200, Abcam; for OCN, 1:200, R\&D; for perilipin, 1:400, Sigma), and incubated them with secondary antibodies.

\section{Cell Isolation and Culture}

We euthanized the mice and dissected the bilateral femurs as well as tibias to collect the marrow cells. Then we planted the cells in Dulbecco's modified Eagle's medium (DMEM) with fetal bovine serum (FBS, 12\%). BMSCs at passage three were incubated with the antibody of CD34, CD45, CD29, and CD90 (Thermo Fisher Scientific, United States) in phycoerythrin and sorted by flow 


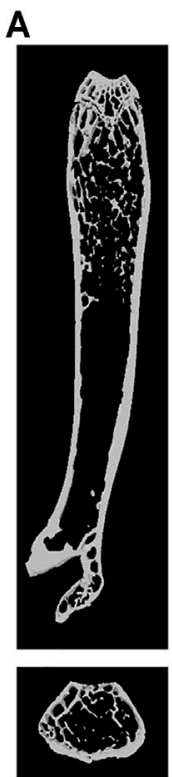

3 Mon

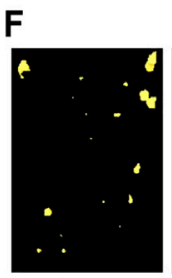

3 Mon
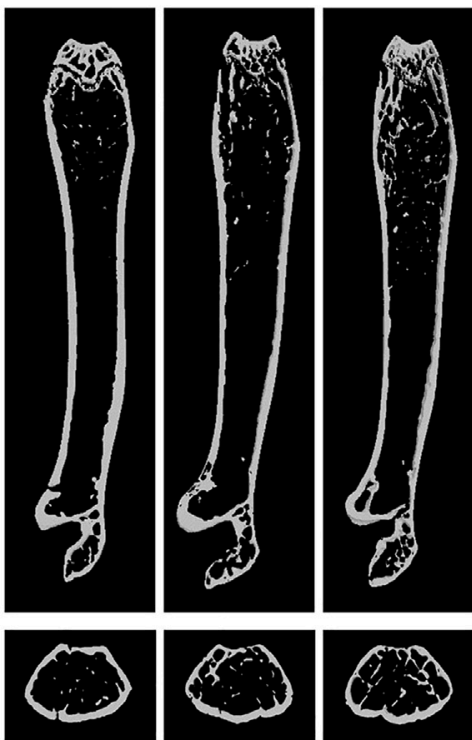

18 Mon

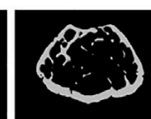

50mg CCG 100mg CCG

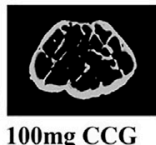

D
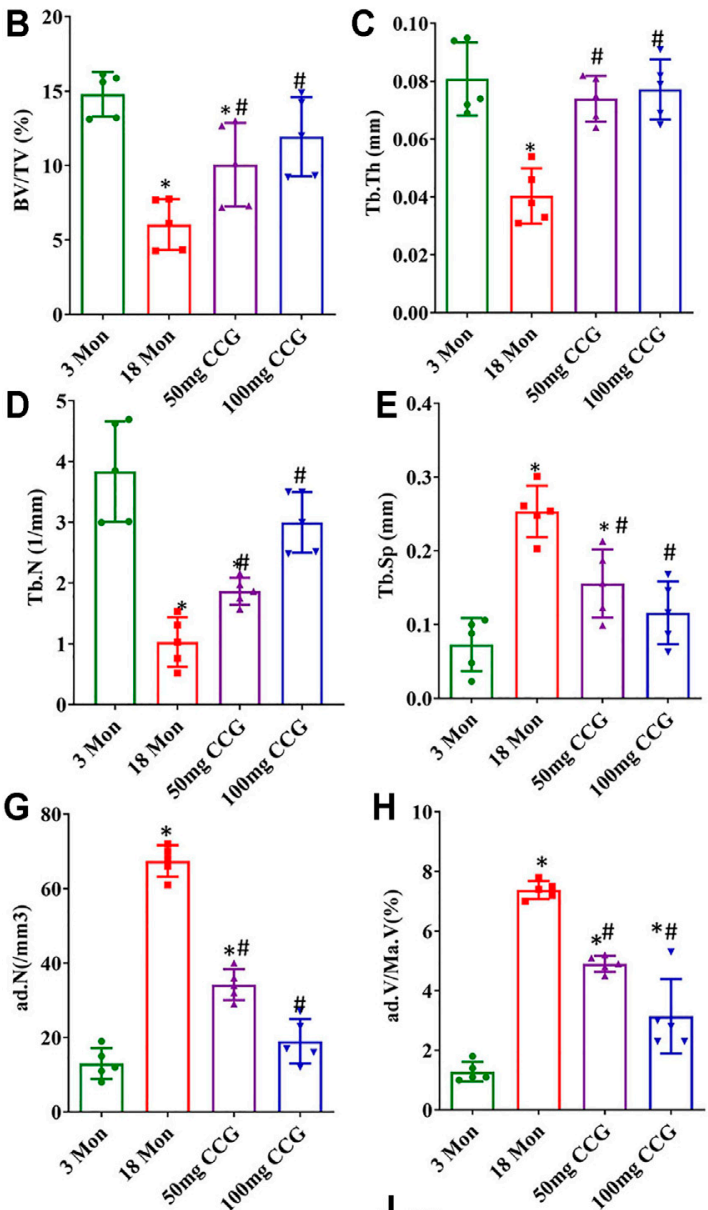

I

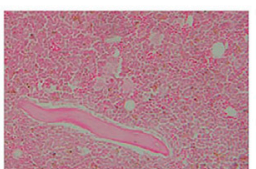

3 Mon

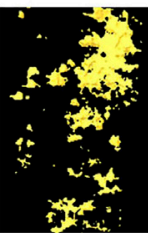

18 Mon

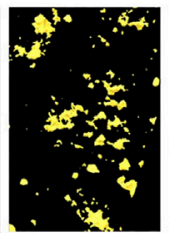

$50 \mathrm{mg}$ CCG 100mg CCG

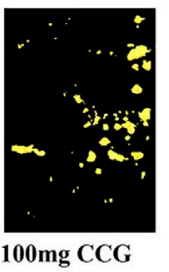

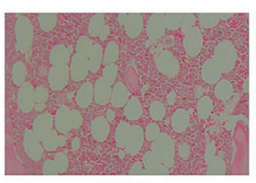

18 Mon

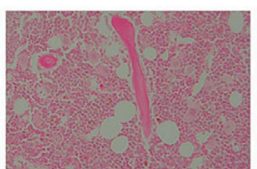

50mg CCG

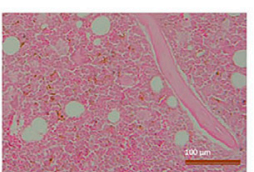

$100 \mathrm{mg} \mathrm{CCG}$

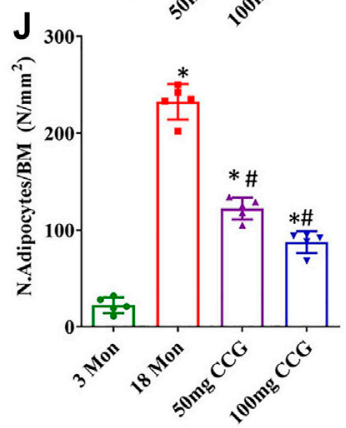

FIGURE 1 | CCG ameliorated bone loss and marrow adiposity of aging mice. (A-E) Representative $\mu$-CT images of femurs from the animals in different groups to acquire the quantitative analysis of trabecular bone. (F-H) Representative $\mu$-CT images of $\mathrm{OsO}_{4}$ staining of femurs from the animals in different groups to acquire the quantitative analysis of the volume of adipocytes. $(\mathbf{I}, \mathbf{J})$ Representative images of H\&E staining to show the ratio of adipocyte number to the area of bone marrow. Scale bar: $100 \mu \mathrm{m}(\mathrm{n}=5){ }^{*} p<0.05$ vs. the 3-mon mice group; \#p < 0.05 vs. the 18-mon mice group.

cytometry by different cell fluorescence for identification. To identify the effect of CCG on cell cycles, 3 days treated cells were harvested and fixed with 70\% ethanol. After washing in PBS, cells were stained with propidium iodide (Sigma, United States) (5 mg/ $\mathrm{ml}$ ) for $30 \mathrm{~min}$ in the dark at $4^{\circ} \mathrm{C}$. Fluorescence was measured with the flow cytometer equipped with a 570-nm argon ion laser (Epics XL, Beckman Coulter Corporation, FL) and the data were analyzed using the Muticycle AV software.

\section{Cell Differentiation}

For one thing, we mixed $\beta$-glycerophosphoric acid $(10 \mathrm{mM}$, Sigma), dexamethasone (10 nM, Sigma), and ascorbic acid $(50 \mu \mathrm{g} / \mathrm{ml}$, Sigma $)$ with the growth medium to induce osteogenesis. For another thing, we added dexamethasone (1 $\mu \mathrm{M}$, Sigma), insulin $(10 \mathrm{mg} / \mathrm{ml}$, Sigma $)$, and methyl isobutylxanthine $(500 \mathrm{mM}$, Sigma) to the growth medium to induce adipogenesis. 


\section{CCG Administration In Vitro}

We sub-cultured the cells that reached approximately $80 \%$ confluence into a new flask and replaced the growth medium with differential medium in the absence or presence of CCG (10, 100 , or $1000 \mu \mathrm{M})$. Then, we selected an appropriate concentration of CCG for the next research part.

\section{Cell Viability Assay}

The cells were incubated with MTT ( $5 \mathrm{mg} / \mathrm{ml}$, Solarbio) to form crystals. We then added dimethyl sulfoxide (DMSO, Solarbio) to fully dissolve the crystals in $10 \mathrm{~min}$. At a wavelength of $490 \mathrm{~nm}$, we measured the absorbance by using the microplate spectrophotometer (BioTek Instruments, United States) to acquire the cell viability assay.

\section{Plasmid Transfection}

We used the plasmid mixed with small interfering RNA sequences for gene knockdown research. The designed and synthesized SiTAZ was used to knock down the TAZ expression (Genechem, China). We used the Lipofectamine 3000 (Thermo Fisher Scientific) for transfection. After $24 \mathrm{~h}$, the transfected cells were ready for the subsequent experiments. Furthermore, we measured the transfection efficiency using flow cytometry (Epics XL, Beckman Coulter Corporation, United States). The sequences of SiTAZ were:

\section{5'-GATCCCCTGGACCAAGTATATGAACCACTCGAG TGGTTCATATACTTGGTCCAGTTTTTGGAT-3'; 5'-AGCTATCCAAAAACTGGACCAAGTATATGAACC ACTCGAGTGGTTCATATACTTGGTCCAGGG-3' .}

\section{Alizarin Red Staining}

We conducted the AR-S after 14-days treatment of osteogenic cocktail. We washed the cells and fixed them with $4 \%$ paraformaldehyde. Then we incubated the washed cells with AR $(0.1 \%$, Sigma). Then, the cells were de-stained with $10 \%$ cetylpyridinium chloride (Sigma) for AR quantification. We detected the $\mathrm{OD}$ value at $562 \mathrm{~nm}$ wavelength to calculate the calcium concentrations.

\section{Oil Red O Staining}

We conducted the oil red $\mathrm{O}$ staining after 14-days treatment of the adipogenic medium. The cells were then incubated with oil red O solution ( $0.5 \%$, Sigma). After fully washed, we acquired the staining images using the microscope. Then we de-stained the treated cells with isopropanol in PBS for quantification study. We detected the $O D$ value at $520 \mathrm{~nm}$ wavelength to calculate the lipid droplets using the microscope (Leica).

\section{Real-Time Reverse Transcription-Polymerase Chain Reaction} We extracted the total RNA using TRIzol ${ }^{\circledR}$ reagent (Ambition, United States). A total of $1 \mu \mathrm{g}$ RNA was reversed-transcribed by using cDNA synthesis Kit (Thermo Fisher Scientific). Real-time RTPCR was performed on a CFX96 Real-Time PCR Detection System (Bio-Rad, Hercules, CA) using SuperReal PreMix Plus (TIANGEN, Beijing, China). All primers were synthesized by Invitrogen
(Carlsbad, CA). The relative expression of mRNAs was calculated according to the ratio of the copy numbers of the target genes (TAZ, RUNX2, OCN, PPAR $\gamma$, perilipin) to the housekeeping gene GAPDH in each sample. The relative gene expression values were evaluated by the 2- $\triangle \triangle \mathrm{Ct}$ method [24]. Forward and reverse primers are listed in Supplementary Table S1.

\section{Western Blotting Analysis}

We isolated the proteins from the treated cells seeded in $60-\mathrm{mm}$ plastic dishes. We separated the target proteins using $12 \%$ sodium dodecyl sulfate polyacrylamide gel electrophoresis (SDS-PAGE; Costar) and transferred them into a polyvinylidene fluoride (PVDF; Costar) membrane. Membranes were blocked with 5\% milk and incubated with primary antibodies against TAZ (1:200; Abcam), RUNX2 (1:200; Cell Signaling), OCN (1:200; Takara Bio), PPAR $\gamma$ (1:100; Cell Signaling), perilipin (1:400; Abcam), p-ERK (1:100; Cell Signaling), ERK (1:100; Cell SignalingA), or GAPDH (1:200, Bioworld, United States). Then we induced the conjugated secondary antibody (IRDye $800^{\circledR}, 1: 2000$, Rockland, United States) and scanned the samples using the Odyssey Infrared Imaging System (Li-COR Biosciences, Beijing, China). ImageJ (version 1.46) was used to determine the integrated intensity of the detected band (Tan and Dai, 2019).

\section{Statistics}

Quantitative results were expressed as mean \pm standard deviation (SD). In vivo, there were five mice in each group; in vitro, all experiments were replicated at least three times. Independent samples $t$-test for the comparison of two groups, one-way analysis of variance (ANOVA) followed by Student Newman Keuls (S-N$\mathrm{K})$ post hoc analysis for the parametric data among multiple groups, which were performed by using SPSS (v.21.0). Values were considered statistically significant at $p<0.05$.

\section{RESULTS}

\section{CCG Ameliorated Bone Loss and Reduced Marrow Adiposity of Aging Mice}

We used $\mu \mathrm{CT}$ to analyze the trabecular bone structure as well as the marrow adipose tissue volume. The BV/TV, Tb.Th, and Tb.N of the femur bone significantly decreased in the 18-mon mice relative to the 3-mon mice, and the $\mathrm{Tb}$. $\mathrm{Sp}$ in marrow significantly increased in the 18-mon mice compared with the 3-mon mice (Figures 1A-E). Simultaneously, fat was significantly accumulated with aging, which was demonstrated by more lipid droplets stained with osmium tetroxide $\left(\mathrm{OsO}_{4}\right)$ in the marrow (Figures 1F-H). After oral administration with $50 \mathrm{mg} / \mathrm{kg} / \mathrm{d}$ or $100 \mathrm{mg} / \mathrm{kg} / \mathrm{d}$ CCG for 2 months, the age-related bone loss and fat accumulation by aging were markedly reduced. First, the $\mu \mathrm{CT}$ results showed an increased bone formation compared with the 18-mon group (Figures 1A-E). Second, a significantly lower amount of fat droplets appeared in $50 \mathrm{mg} / \mathrm{kg} / \mathrm{d}$ or $100 \mathrm{mg} / \mathrm{kg} / \mathrm{d}$ CCG administration group (Figures $\mathbf{1 F}-\mathbf{H}$ ). Similarly, H\&E staining calculated a lower number of adipocytes after oral administration of CCG (Figures 1I,J). These data suggested that oral administration of CCG 


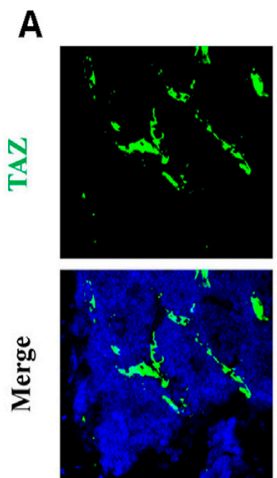

3 Mon
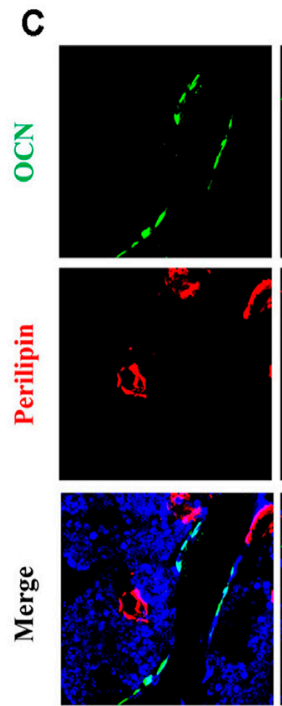

3 Mon

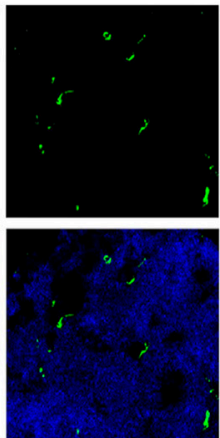

18 Mon
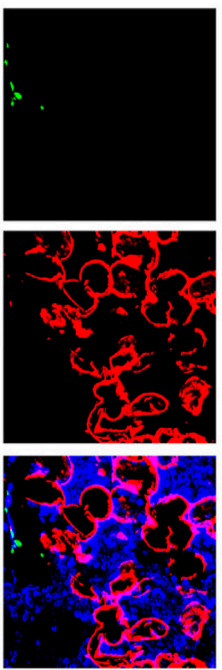

18 Mon
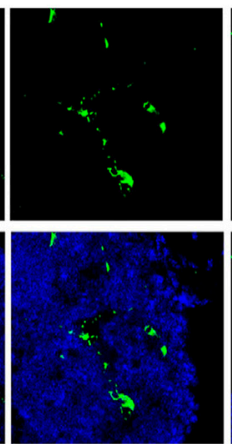

50mg CCG
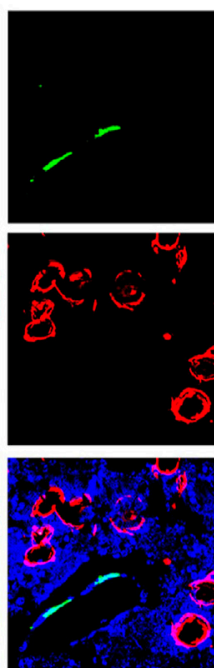

50mg CCG
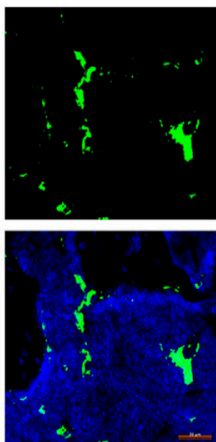

100mg CCG
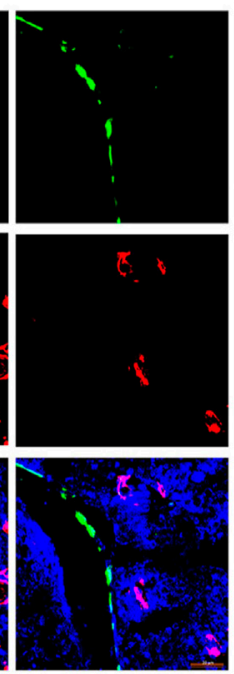

$100 \mathrm{mg}$ CCG
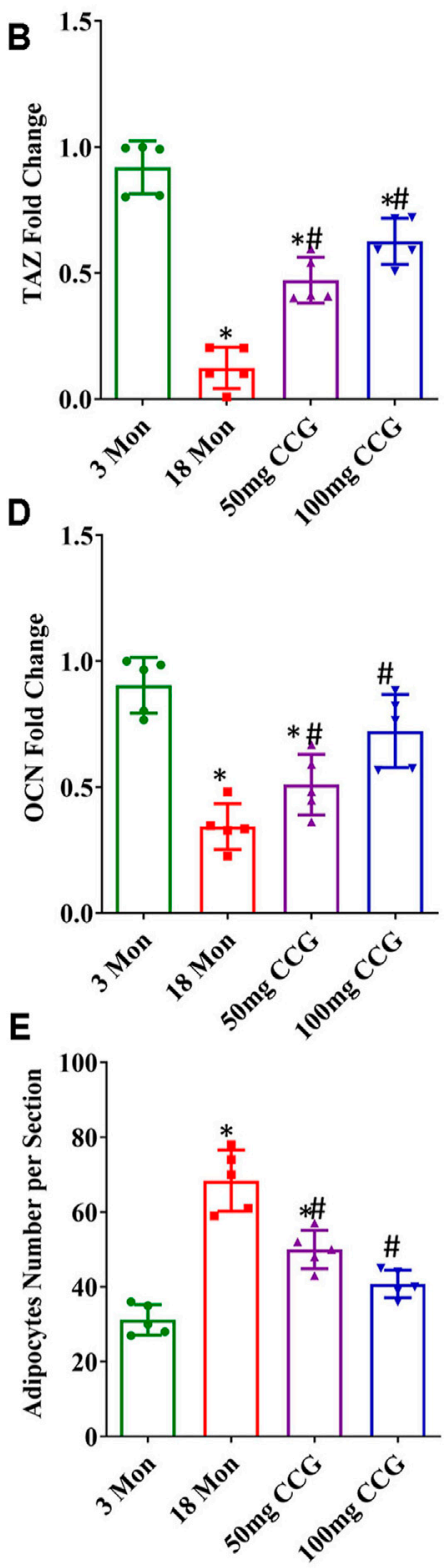

FIGURE 2 | CCG targeted TAZ to regulate the switch from adipogenesis to osteogenesis of BMSCs in vivo. (A, B) Representative images and quantitative analysis of TAZ (green) expression in the mice femurs from different groups. (C-E) Immunofluorescent staining of OCN (green) and perilipin (red) in the mice femurs and the relevant quantitative analysis. Scale bar: $50 \mu \mathrm{m}(\mathrm{n}=5){ }^{*} p<0.05$ vs. the 3-mon mice group; \#p < 0.05 vs. the 18 -mon mice group.

ameliorated bone loss and marrow adiposity of aging mice. Furthermore, CCG was demonstrated to inhibit osteoclasts formation by TRAP staining (Supplementary Figure S1).

CCG Increased TAZ Expression to Regulate Switch from Adipogenesis to Osteogenesis in Bone Marrow

TAZ expression was decreased with aging, while $50 \mathrm{mg} / \mathrm{kg} / \mathrm{d}$ or $100 \mathrm{mg} / \mathrm{kg} / \mathrm{d}$ dose of CCG offset the age-related down-expression of TAZ (Figures 2A,B). Consequently, we found a decreased expression of osteocalcin (OCN) (the marker for osteogenesis) and an increased expression of perilipin (the marker for adipogenesis) in aging mice (Figures 2C-E). CCG administration ameliorated the down-expression of OCN and decreased the up-expression of perilipin in aging mice (Figures 2C-E). Above all, we speculated that CCG targeted TAZ to regulate the switch from adipocytes to osteoblasts of BMSCs. In order to confirm our hypothesis, we conducted experiments in vitro study. 


\section{CCG Targeted TAZ to Facilitate Osteogenic Differentiation of BMSCs In Vitro}

The hematopoietic surface markers CD34, CD45, CD29, and CD90 were detected to identify the BMSCs (Supplementary Figure S2). Then, we performed MTT assays and found that the cell viabilities were decreased after $1000 \mu \mathrm{M}$ CCG treatment but increased after administration with $100 \mu \mathrm{M}$ of CCG. Of interest, $10 \mu \mathrm{M}$ CCG did not influence the cell viability (Figure 3A). To verify whether the administration of $100 \mu \mathrm{M}$ CCG influenced the proliferation of BMSCs, we calculated the percentage of the cells in the G1 phase with the flow cytometer after 3 days' treatment. As a result, we found that the percentage of the cells in the G1 phase did not change after the CCG treatment (Supplementary Figure S3).

In order to confirm whether CCG had anti-aging effects, we identified the expression of p21 and p16 using real-time RT PCR analyses. We found that the CCG treatment induced the downexpression of p21 and p16 (Supplementary Figure S4). Further, CCG was proved to facilitate the most osteogenesis at the concentration of $100 \mu \mathrm{M}$. On one hand, $100 \mu \mathrm{M}$ of CCG accelerated the calcium deposition evidenced by AR-S experiment (Figures 3B,C). On the other hand, the highest mRNA levels of RUNX2 and OCN expressed in $100 \mu \mathrm{M}$ CCG group (Figures 3D,E). Thus, $100 \mu \mathrm{M}$ CCG was administrated on cells in the subsequent experiments. Inevitably, $100 \mu \mathrm{M}$ of CCG markedly increased the expression of TAZ, RUNX2, and OCN on Day 3, 7, and 14 (Figures 3F-I). Meanwhile, the adipogenic markers were down-regulated during osteogenesis by CCG treatment (Supplementary Figure S5A). Then, the TAZ expression was knocked down by transfecting with plasmids containing SiTAZ sequences (Figures 3J-L). SiTAZ significantly offset the CCG induced up-expression of the markers for osteogenesis, RUNX2, and OCN (Figures 3M-Q). Moreover, the AR-S results suggested SiTAZ delayed the recruitment of osteogenic nodules by CCG (Figures 3R, S). Above all, CCG might facilitate osteogenesis by targeting TAZ with a peak at the concentration of $100 \mu \mathrm{M}$ for BMSCs differentiation.

\section{CCG Targeted TAZ to Reduce Adipogenic Differentiation of BMSCs In Vitro}

As well, we performed MTT assays and found that the cell viabilities were decreased after $1000 \mu \mathrm{M}$ CCG administration but increased after administration with $100 \mu \mathrm{M}$ of CCG during adipogenic differentiation of BMSCs, and $10 \mu \mathrm{M}$ CCG did not influence the cell viability (Figure 4A). The Oil Red O staining results demonsrated that $100 \mu \mathrm{M}$ of CCG decreased the lipid droplets during adipogenesis on Day 14 (Figures 4B,C). Consistently, the lowest mRNA levels of PPAR $\gamma$ and perilipin but the highest TAZ expression appeared in $100 \mu \mathrm{M}$ CCG group during adipogenesis (Figures 4D,E). Meanwhile, the osteogenic markers were up-regulated during the administration of CCG treatment (Supplementary Figure S5B). Thus, $100 \mu \mathrm{M}$ CCG was also administrated on cells in the subsequent experiments during adipogenesis. Inevitably, the expression of PPAR $\gamma$ and perilipin was markedly decreased and the TAZ protein levels were significantly increased by $100 \mu \mathrm{M}$ CCG administration during adipogenesis (Figures 4F-I). Then the TAZ expression was knocked down by transfecting with plasmids containing SiTAZ sequences (Figures 4J-L). Both at the mRNA and protein levels, TAZ knockdown markedly lessened the CCG induced down-expression of PPAR $\gamma$ and perilipin (Figures 4M-Q). Accordingly, the Oil Red O staining also points a link between CCG treatment and TAZ signaling transduction during adipogenesis of BMSCs (Figures $4 \mathbf{R}, \mathbf{S}$ ). Taken together, we hypothesized that CCG targeted TAZ to reduce marrow adiposity in vitro.

\section{CCG Activated p-ERK Signal to Modulate TAZ Expression and BMSCs Lineage Commitment}

To elucidate the signaling transduntion, we conducted inhibitor study using pathway inhibitors. We found that the up-expression of TAZ induced by CCG administration was significantly diminished by UO126, rather than LY294002 (Figures 5A-C). Furthermore, the p-ERK/ERK ratio could be augmented by CCG, suggesting CCG could stimulate the MEK-ERK signaling transduction during osteogenesis (Figures 5D-F). Additionally, the AR-S results reflected that UO126 attenuated the formation of osteogenic nodules accelerated by CCG (Figures 5G,H). Similar results were confirmed during adipogenesis to that during osteogenesis (Figure 6). We concluded that the MEK-ERK signaling pathway was involved in the CCG-TAZ axis to facilitate osteogenesis at the expense of adiposity in bone marrow for anti-aging related osteoporosis.

\section{DISCUSSION}

Osteoporosis is recognized as a common concern in the aging population worldwide (Curtis et al., 2015). Osteogenesis reduction and fat accumulation triggered an imbalance of bone remodeling (Infante and Rodríguez, 2018). With aging progressing, senile osteoporosis becomes a growing public problem. The importance of developing new antiosteoporosis therapies targeted at osteoblasts not only to increase bone formation and induce bone growth, but also to prevent age-related fat accumulation (Li et al., 2015). Increasing evidences bestow Chinese herbs vital roles in the therapy of osteoporosis and bone fracture, with Curculigo orchioides identified as widely used in senile osteoporosis (Wang et al., 2012; Wu et al., 2012; Tan et al., 2019). The potential biological functions and molecular mechanisms of the effects of CCG, the major bioactive component of Curculigo orchioides, on the switch from adipocytes into osteoblasts in aging mice has never been reported.

In our present study, the $\mu \mathrm{CT}$ analyses revealed that oral administration of CCG ameliorated the bone loss and marrow adiposity in the bone of aging mice. Previously, researchers 


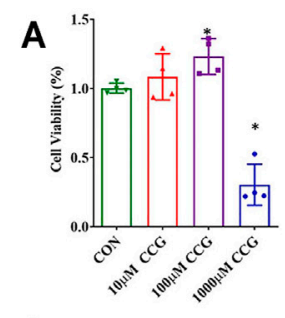

C
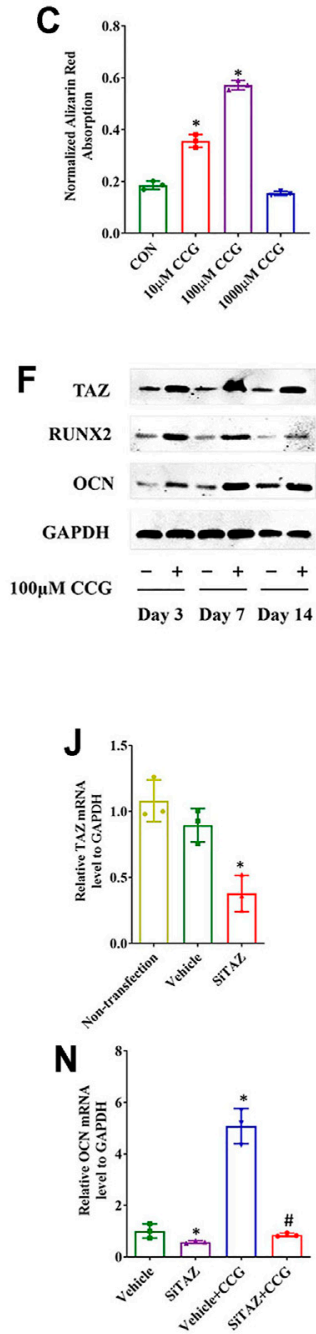

$\mathbf{R}$

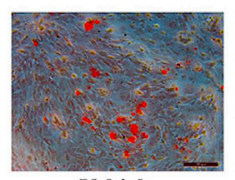

Vehicle

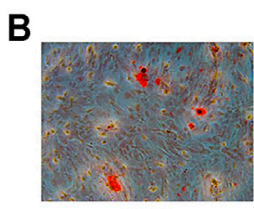

CON

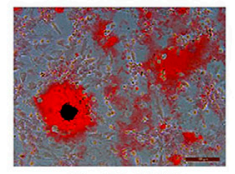

$10 \mu \mathrm{M} \mathrm{CCG}$

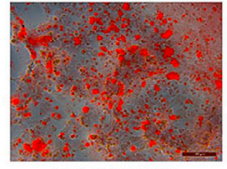

$100 \mu \mathrm{M}$ CCG

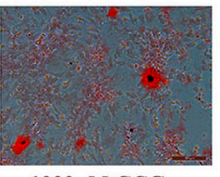

$1000 \mu \mathrm{M}$ CCG
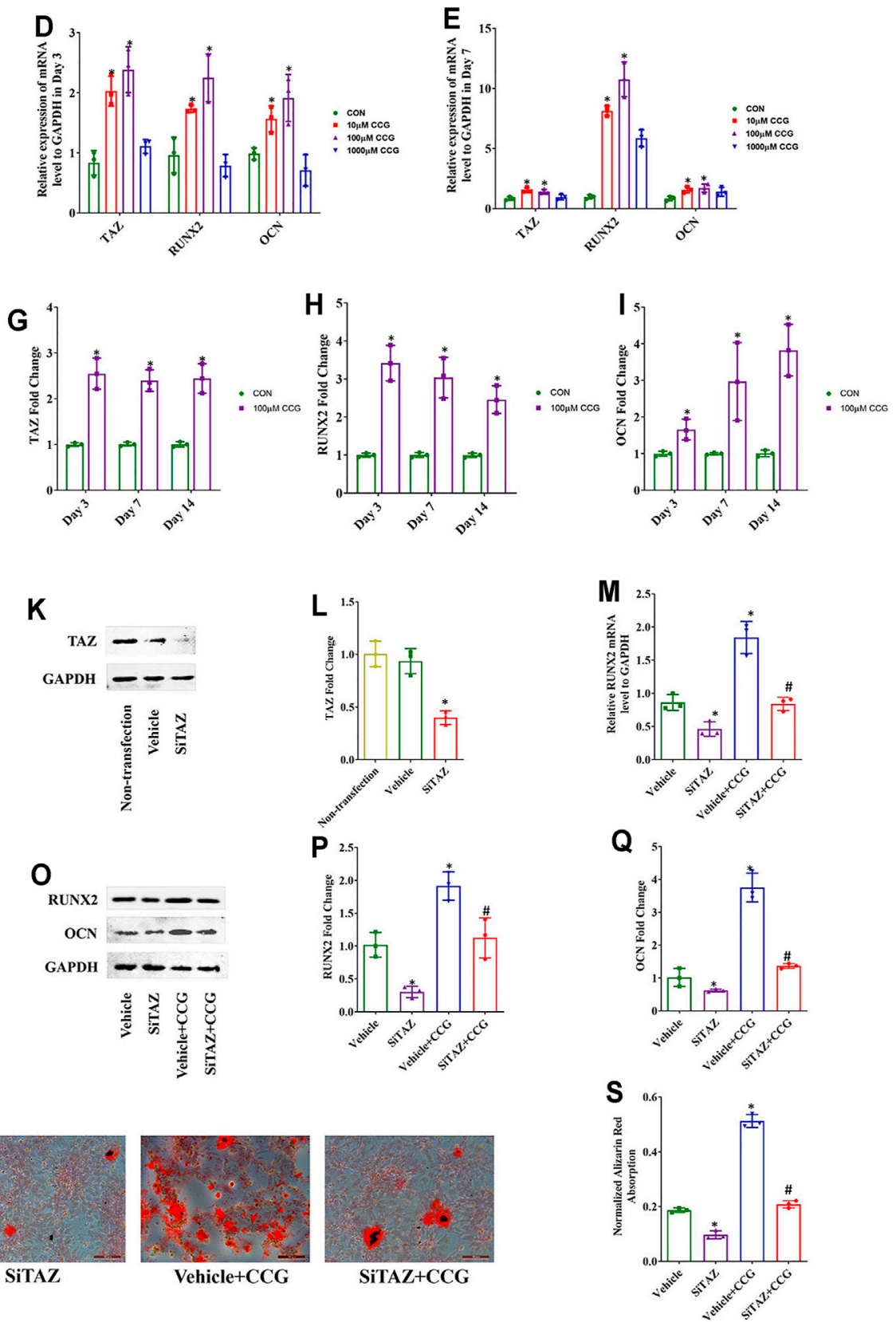

FIGURE 3 | CCG targeted TAZ to facilitate osteogenic differentiation of BMSCs in vitro. (A) MTT assays presented cell viabilities after CCG administration during osteogenesis of BMSCs. (B, C) Representative images of AR-S results in different mice groups. Scale bar: $100 \mu \mathrm{m}$. (D, E) Relative mRNA levels of TAZ, RUNX2, and OCN with or without CCG treatment. (F-I) TAZ, RUNX2, and OCN expression in protein levels with or without CCG treatment. ${ }^{*}<0.05$ vs. the control group. (J-L) SiTAZ plasmid significantly knocked down the mRNA and protein levels of TAZ during the osteogenesis of BMSCs. (M-Q) SiTAZ discounted the CCG induced upregulation of RUNX2 and OCN. (R, S) Representative images of AR-S showed the differences of calcium deposits in different groups. Bar graphs showed the means \pm SD from three independent experiments. Scale bar: $100 \mu \mathrm{m}(\mathrm{n}=3){ }^{*} p<0.05$ vs. the Vehicle group; \#p $<0.05$ vs. the Vehicle + CCG group. 

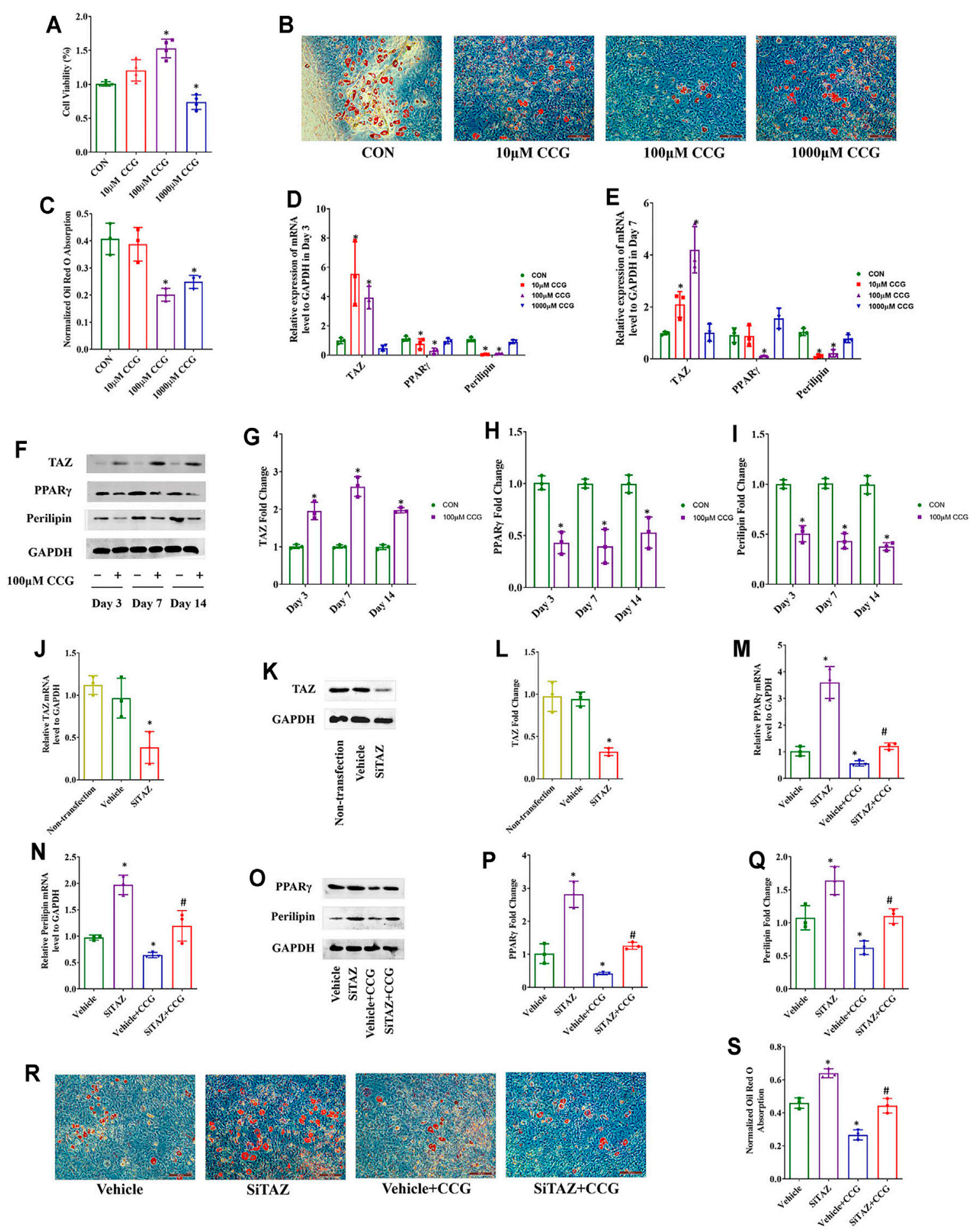

FIGURE 4 | CCG targeted TAZ to reduce adipogenic differentiation of BMSCs in vitro. (A) MTT assays presented cell viabilities after CCG administration during adipogenesis of BMSCs. (B, C) Representative images of Oil Red O staining reflected the lipid droplets. Scale bar: $100 \mu m$. (D, E) Relative mRNA levels of TAZ, PPAR $\gamma$, and perilipin to GAPDH were presented in Day 3 and Day 7 in the absence or presence of CCG during adipogenesis. (F-I) Relative protein levels of TAZ, PPAR $\gamma$, and perilipin to GAPDH were presented in Day 3, in Day 7, and in Day 14 after the treatment. ${ }^{*} p<0.05$ vs. the control group. (J-L) Relative mRNA and protein levels to GAPDH of TAZ were significantly knocked down by the SiTAZ plasmid during adipogenesis. (M-Q) SiTAZ discounted the CCG induced up-expression of TAZ and down-expression of PPAR $y$ and perilipin at Day 3 after the treatment. $(\mathbf{R}, \mathbf{S})$ Representative images of Oil Red O staining showed the lipid droplets in different groups. Bar graphs showed the means \pm SD from three independent experiments. Scale bar: $100 \mu \mathrm{m}(n=3)^{*} p<0.05$ vs. the Vehicle group; \#p $<0.05$ vs. the Vehicle $+C C G$ group. 

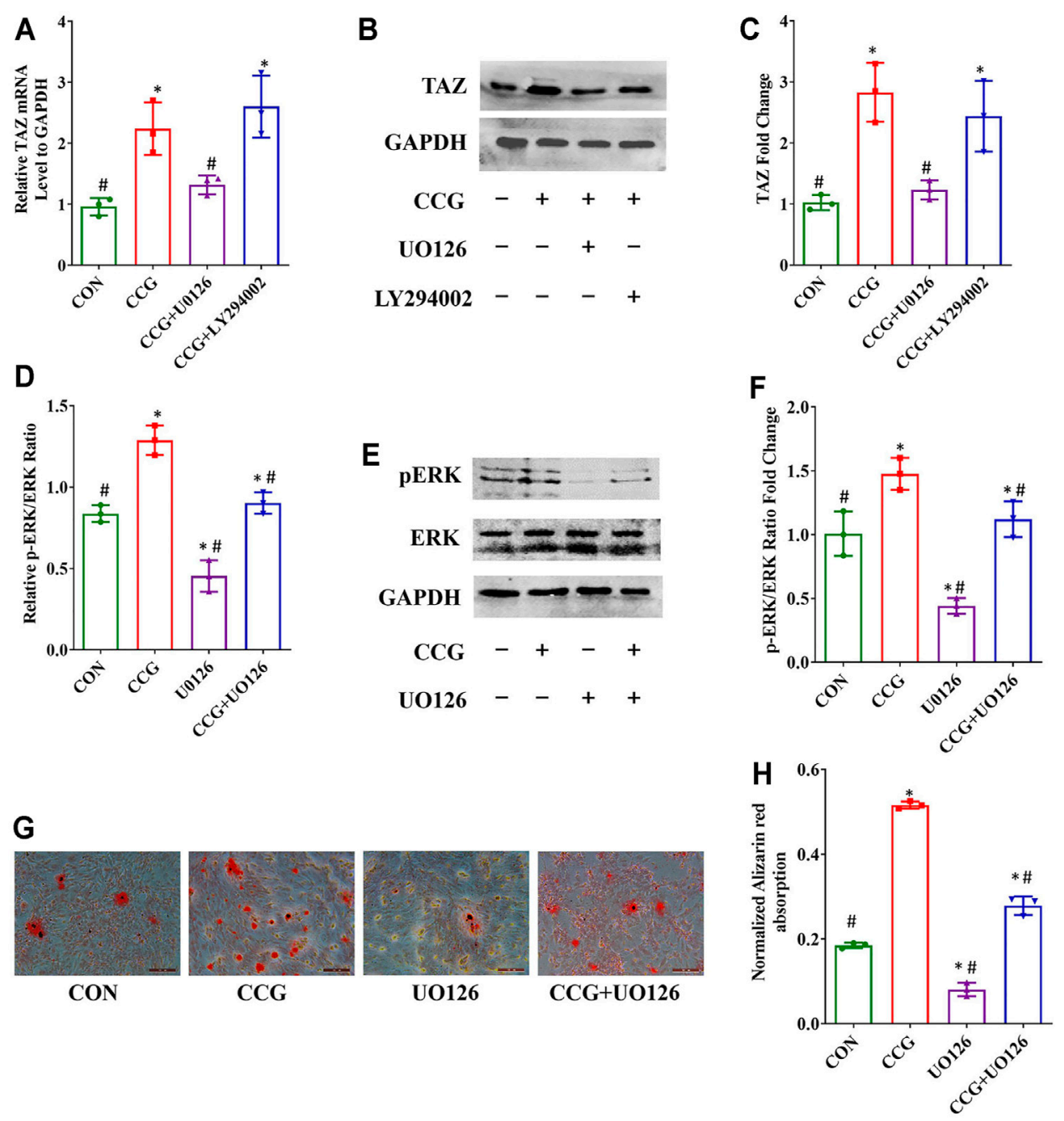

FIGURE 5 | Osteogenic differentiation and TAZ expression were increased by CCG treatment by increasing the p-ERK/ERK ratio. (A-C) Relative mRNA and protein levels to GAPDH of TAZ at Day 3 in different groups. (D-F) Relative mRNA and protein levels of p-ERK at Day 3 in different groups. (G, H) Representative images of AR-S showed the calcium deposits were influenced by CCG treatment. Scale bar: $100 \mu \mathrm{m}{ }^{*} p<0.05$ vs. the control group (cells cultured in osteogenic medium during osteogenesis); \#p < 0.05 vs. the CCG administration group.

reported that CCG prevented bone loss in ovariectomized rats (Shen et al., 2013). Consistently, other researchers have demonstrated that CCG stimulated the secretion of bone morphogenetic protein 2 (BMP-2) to promote osteogenesis (Ma et al., 2011). As was reported, CCG also prevented oxidative damage and inhibited osteoclastogenesis in rat bone marrow cells (Wang et al., 2012). As well, our study provided new insight into the molecular mechanisms how CCG regulates the age-related adipocytes-osteoblasts lineage commitment through MEK/ERK-TAZ axis. Thus, CCG might be a good candidate for further development as an antiosteoporotic remedy in an aging population.

Latent bone-lining osteoblast-adipocyte precursors, including BMSCs, are activated in response to various stimuli including exogenous and endogenous changes causing pre-osteoblasts to increase surface expression of osteogenic markers at the expense of reduced adipogenic markers (Paspaliaris and Kolios, 2019). In addition, the microenvironment significantly affects bone mass by the regulation of vital factors ( $\mathrm{Du}$ et al., 2019). For instance, TAZ exerted pivotal effects on stem cell fate determination (Panciera et al., 2017; Salem and Hansen, 2019; Zheng and Pan, 2019). In our previous study, we confirmed TAZ as an important transcriptional modulator during osteoblastogenesis, which was evoked by insulin-like growth factor 1 (IGF-1) (Xue et al., 2013). In the present study, we confirmed that the TAZ signaling was involved in the regulation of the differential shift from adipogenesis to osteogenesis by CCG. In vivo, the down-expression of TAZ in aging mice was ameliorated by the oral administration of CCG with up-expression of osteogenic genes and down-expression of adipogenic genes. In vitro, TAZ knockdown could offset CCG-regulated expression of osteogenic genes and adipogenic genes. Hereby, our results suggested that CCG influenced the 

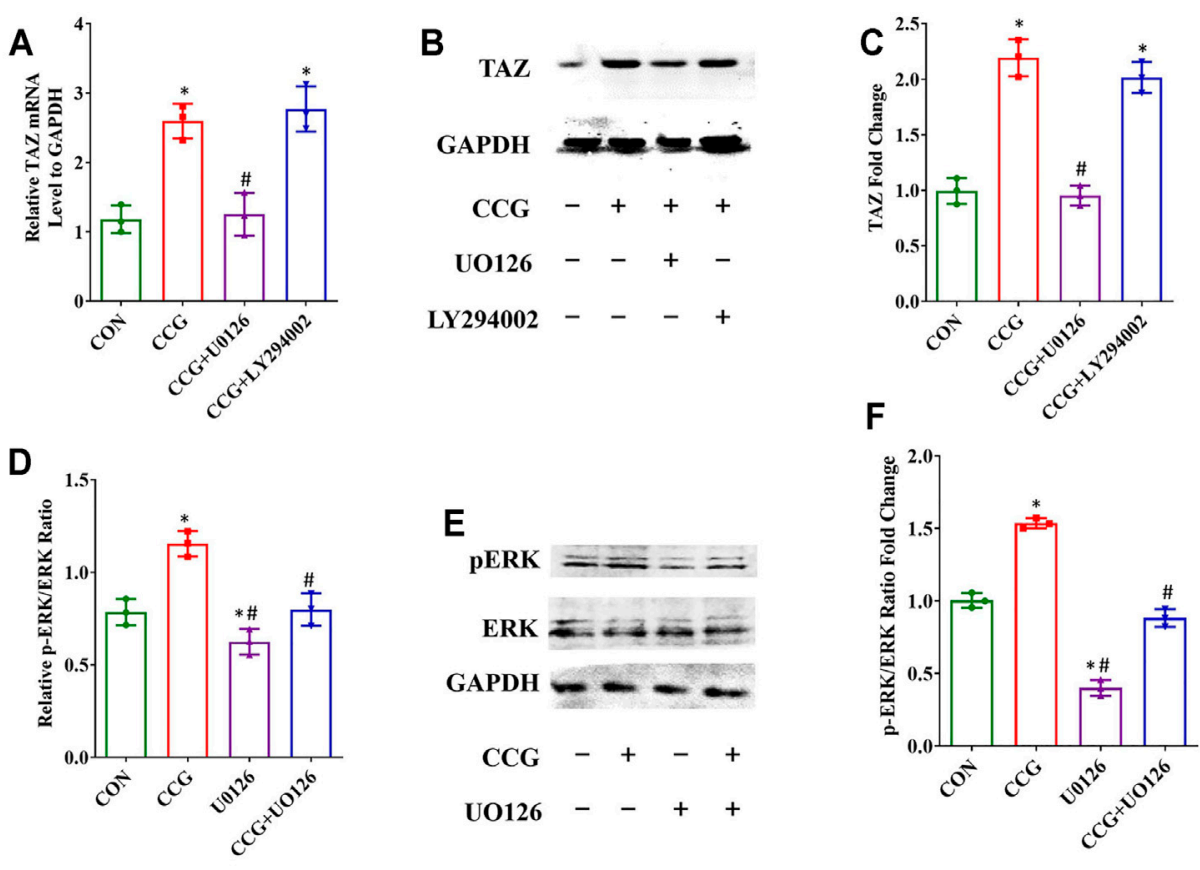

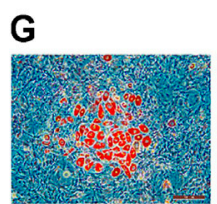

CON

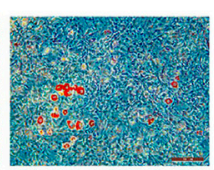

CCG
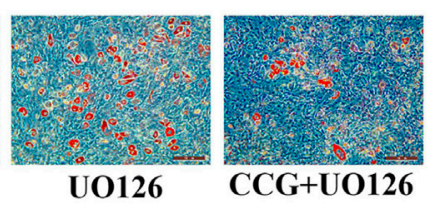

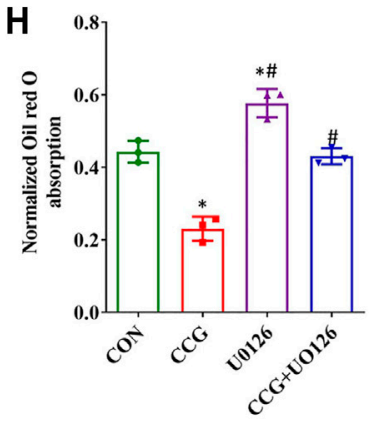

FIGURE 6 | Decreased adipogenic differentiation accompanied by increased TAZ expression was also mediated by p-ERK/ERK signaling after CCG treatment. (A-C) Relative mRNA and protein levels of TAZ to GAPDH at Day 3 after the treatment during adipogenesis. (D-F) Relative mRNA and protein levels of p-ERK at Day 3 after the treatment during adipogenesis. (G, H) Representative images of Oil Red O staining showed the lipid droplets were lessened by CCG. Scale bar: 100 um $(n=3)$ ${ }^{*} p<0.05$ vs. the control group (cells cultured in adipogenic medium during adipogenesis); \#p < 0.05 vs. the CCG treatment group.

age-related switch of cells lineage commitment of BMSCs via TAZ signal pathway.

As recognized commonly, mitogen activated protein kinases (MAPKs)-mediated signaling pathways pronounced markedly in cell differentiation and proliferation (Elango et al., 2019; Kim et al., 2019). Differential molecular in signaling pathways goes through the plasma membrane from extracellular to intracellular environment, then regulates the osteogenesis and adipogenesis (Husain and Jeffries, 2017; Wu et al., 2017; Bukowska et al., 2018). In the early 1990s, as one of the members of the MAPK family, the MEK-ERK pathway was first recognized (Xu et al., 2016). The activation of p-ERK had been shown to be essential for RUNX2 up-regulation and transcriptional activity previously (Li et al., 2017). As was proved, TAZ directly combined to RUNX2 to promote osteogenesis (Wang et al., 2019b). Previously, our study also pointed a molecular link of
MEK-ERK pathway to the TAZ during BMSCs osteogenesis (Xue et al., 2013; Wang et al., 2019a; Tan and Dai, 2019). Here, we found that CCG up-regulated TAZ signaling via the MEKERK pathway, which might be identified as the underlying regulation network. The MEK-ERK pathway was activated upon CCG-mediated osteogenic differentiation of BMSCs, while the MEK-ERK inhibitors, UO126 reduced the CCGmediated calcium deposits during cell differentiation. These findings suggested that MRK-ERK activation was the upstream signal by which CCG-induced TAZ up-expression and then regulated the cell differentiation.

\section{CONCLUSION}

Our present study confirmed that CCG ameliorated agerelated bone loss by upregulating TAZ expression to induce 


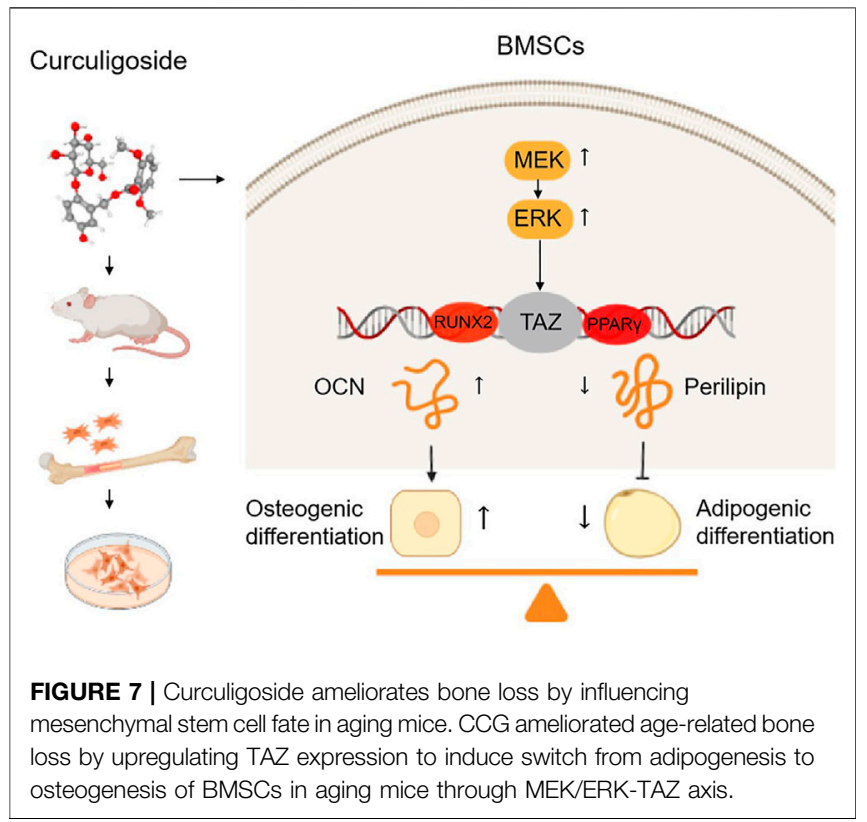

the switch from adipogenesis to osteogenesis of BMSCs in aging mice through $\mathrm{MEK} / \mathrm{ERK}-\mathrm{TAZ}$ axis. In vivo, oral administration of CCG significantly increased the bone mass and decreased the adipocyte numbers in the bone marrow of aging mice. In vitro, a proper concentration of CCG regulated TAZ expression through the MEK-ERK pathway to facilitated osteogenesis at the expense of reduced adipogenesis of BMSCs (Figure 7).

All these results revealed that CCG targeted TAZ to ameliorate bone loss in aging mice, which provided the evidence for the clinical use in age-related osteoporosis. In summary, our study pointed the new link of CCG to TAZ signaling and provided a novel therapeutic target for senile osteoporosis.

\section{REFERENCES}

Ambrosi, T. H., Scialdone, A., Graja, A., Gohlke, S., Jank, A.-M., Bocian, C., et al. (2017). Adipocyte Accumulation in the Bone Marrow during Obesity and Aging Impairs Stem Cell-Based Hematopoietic and Bone Regeneration. Cell Stem Cell 20, 771-784. doi:10.1016/j.stem.2017.02.009

Bukowska, J., Frazier, T., Smith, S., Brown, T., Bender, R., McCarthy, M., et al. (2018). Bone Marrow Adipocyte Developmental Origin and Biology. Curr. Osteoporos. Rep. 16, 312-319. doi:10.1007/s11914-018-0442-z

Byun, M. R., Lee, C. H., Hwang, J.-H., Kim, A. R., Moon, S. A., Sung, M. K., et al. (2013). Phorbaketal A Inhibits Adipogenic Differentiation through the Suppression of PPAR $\gamma$-Mediated Gene Transcription by TAZ. Eur. J. Pharmacol. 718, 181-187. doi:10.1016/j.jphar.2013.08.035

Cao, D. P., Zheng, Y. N., Qin, L. P., Han, T., Zhang, H., Rahman, K., et al. (2008). Curculigo Orchioides, a Traditional Chinese Medicinal Plant, Prevents Bone Loss in Ovariectomized Rats. Maturitas 59, 373-380. doi:10.1016/ j.maturitas.2008.03.010

Chen, T., Wang, H., Jiang, C., and Lu, Y. (2021). PKD1 Alleviates Oxidative StressInhibited Osteogenesis of Rat Bone Marrow-Derived Mesenchymal Stem Cells through TAZ Activation. J. Cel Biochem. 122 (11), 1715-1725. doi:10.1002/jcb.30124

Curtis, E., Litwic, A., Cooper, C., and Dennison, E. (2015). Determinants of Muscle and Bone Aging. J. Cel. Physiol. 230, 2618-2625. doi:10.1002/jcp.25001

\section{DATA AVAILABILITY STATEMENT}

The raw data supporting the conclusions of this article will be made available by the authors, without undue reservation.

\section{ETHICS STATEMENT}

The animal study was reviewed and approved by the ethics committee of the third hospital of hebei medical university.

\section{AUTHOR CONTRIBUTIONS}

PX and SL designed the research; NW, ZL and SL carried out most of the experiments, generated data, and drafted the manuscript; LG, $\mathrm{XB}, \mathrm{KW}$ and $\mathrm{CL}$ helped to collect the samples; PX, SL and YL supervised the experiments and proofread the manuscript.

\section{FUNDING}

This work was supported by Basic Research Program for BeijingTianjin-Hebei Coordination (No: 19JCZDJC65500Z), Medical application technology program of Hebei Province (No: G2019008), Osteoporosis Program for Young Doctors (No: GX20191107) and Government Foundation to Train Clinical Talents and Leading Specialists (No: 361005).

\section{SUPPLEMENTARY MATERIAL}

The Supplementary Material for this article can be found online at: https://www.frontiersin.org/articles/10.3389/fcell.2021.767006/ full\#supplementary-material

Dalton, S. (2015). Linking the Cell Cycle to Cell Fate Decisions. Trends Cel. Biol. 25, 592-600. doi:10.1016/j.tcb.2015.07.007

Du, Y., Guo, J. L., Wang, J., Mikos, A. G., and Zhang, S. (2019). Hierarchically Designed Bone Scaffolds: From Internal Cues to External Stimuli. Biomaterials 218, 119334. doi:10.1016/j.biomaterials.2019.119334

Elango, J., Robinson, J., Zhang, J., Bao, B., Ma, N., de Val, J. E. M. S., et al. (2019). Collagen Peptide Upregulates Osteoblastogenesis from Bone Marrow Mesenchymal Stem Cells through MAPK- Runx2. Cells 8, 446. doi:10.3390/cells8050446

Hansen, J. B., Petersen, R. K., Larsen, B. M., Bartkova, J., Alsner, J., and Kristiansen, K. (1999). Activation of Peroxisome Proliferator-Activated Receptor $\gamma$ Bypasses the Function of the Retinoblastoma Protein in Adipocyte Differentiation. J. Biol. Chem. 274, 2386-2393. doi:10.1074/jbc.274.4.2386

Hu, L., Yin, C., Zhao, F., Ali, A., Ma, J., and Qian, A. (2018). Mesenchymal Stem Cells: Cell Fate Decision to Osteoblast or Adipocyte and Application in Osteoporosis Treatment. Int. J. Mol. Sci. 19, 360. doi:10.3390/ijms19020360

Huang, L., Wang, X., Cao, H., Li, L., Chow, D. H.-K., Tian, L., et al. (2018). A BoneTargeting Delivery System Carrying Osteogenic Phytomolecule Icaritin Prevents Osteoporosis in Mice. Biomaterials 182, 58-71. doi:10.1016/j.biomaterials.2018.07.046

Husain, A., and Jeffries, M. A. (2017). Epigenetics and Bone Remodeling. Curr. Osteoporos. Rep. 15, 450-458. doi:10.1007/s11914-017-0391-y

Infante, A., and Rodríguez, C. I. (2018). Osteogenesis and Aging: Lessons from Mesenchymal Stem Cells. Stem Cel. Res. Ther. 9, 244. doi:10.1186/s13287-0180995-x 
Jeanette, H., Marziali, L. N., Bhatia, U., Hellman, A., Herron, J., Kopec, A. M., et al. (2021). YAP and TAZ Regulate Schwann Cell Proliferation and Differentiation during Peripheral Nerve Regeneration. Glia 69 (4), 1061-1074. doi:10.1002/ glia.23949

Kegelman, C. D., Mason, D. E., Dawahare, J. H., Horan, D. J., Vigil, G. D., Howard, S. S., et al. (2018). Skeletal Cell YAP and TAZ Combinatorially Promote Bone Development. FASEB j. 32, 2706-2721. doi:10.1096/fj.201700872r

Kim, J. M., Yang, Y. S., Park, K. H., Oh, H., Greenblatt, M. B., and Shim, J. H. (2019). The ERK MAPK Pathway Is Essential for Skeletal Development and Homeostasis. Int. J. Mol. Sci. 20, 1803. doi:10.3390/ijms20081803

Lee, E., Ko, J.-Y., Kim, J., Park, J.-W., Lee, S., and Im, G.-I. (2019). Osteogenesis and Angiogenesis Are Simultaneously Enhanced in BMP2-/VEGF-Transfected Adipose Stem Cells through Activation of the YAP/TAZ Signaling Pathway. Biomater. Sci. 7 (11), 4588-4602. doi:10.1039/c9bm01037h

Li, C.-J., Cheng, P., Liang, M.-K., Chen, Y.-S., Lu, Q., Wang, J.-Y., et al. (2015). MicroRNA-188 Regulates Age-Related Switch between Osteoblast and Adipocyte Differentiation. J. Clin. Invest. 125, 1509-1522. doi:10.1172/jci77716

Li, Y., Ge, C., and Franceschi, R. T. (2017). MAP Kinase-dependent RUNX2 Phosphorylation Is Necessary for Epigenetic Modification of Chromatin during Osteoblast Differentiation. J. Cel. Physiol. 232, 2427-2435. doi:10.1002/jcp.25517

Ma, C., Zhang, J., Fu, J., Cheng, L., Zhao, G., and Gu, Y. (2011). Up-regulation of VEGF by MC3T3-E1 Cells Treated with Curculigoside. Phytother. Res. 25, 922-926. doi:10.1002/ptr.3449

Matsumoto, Y., La Rose, J., Kent, O. A., Wagner, M. J., Narimatsu, M., Levy, A. D., et al. (2016). Reciprocal Stabilization of ABL and TAZ Regulates Osteoblastogenesis through Transcription Factor RUNX2. J. Clin. Invest. 126, 4482-4496. doi:10.1172/jci87802

Muruganandan, S., Govindarajan, R., McMullen, N. M., and Sinal, C. J. (2017). Chemokine-Like Receptor 1 Is a Novel Wnt Target Gene that Regulates Mesenchymal Stem Cell Differentiation. Stem Cells 35, 711-724. doi:10.1002/stem.2520

Panciera, T., Azzolin, L., Cordenonsi, M., and Piccolo, S. (2017). Mechanobiology of YAP and TAZ in Physiology and Disease. Nat. Rev. Mol. Cel Biol. 18, 758-770. doi:10.1038/nrm.2017.87

Paspaliaris, V., and Kolios, G. (2019). Stem Cells in Osteoporosis: From Biology to New Therapeutic Approaches. Stem Cell Int. 2019, 1730978. doi:10.1155/2019/ 1730978

Rosen, C. J., Ackert-Bicknell, C., Rodriguez, J. P., and Pino, A. M. (2009). Marrow Fat and the Bone Microenvironment: Developmental, Functional, and Pathological Implications. Crit. Rev. Eukar Gene Expr. 19 (2), 109-124. doi:10.1615/critreveukargeneexpr.v19.i2.20

Salem, O., and Hansen, C. G. (2019). The Hippo Pathway in Prostate Cancer. Cells 8, 370. doi:10.3390/cells 8040370

Sambrook, P., and Cooper, C. (2006). Osteoporosis. Lancet 367, 2010-2018. doi:10.1016/s0140-6736(06)68891-0

Sanghani-Kerai, A., McCreary, D., Lancashire, H., Osagie, L., Coathup, M., and Blunn, G. (2018). Stem Cell Interventions for Bone Healing: Fractures and Osteoporosis. Cscr 13, 369-377. doi:10.2174/1574888x13666180410160511

Shen, Q., Zeng, D., Zhou, Y., Xia, L., Zhao, Y., Qiao, G., et al. (2013). Curculigoside Promotes Osteogenic Differentiation of Bone Marrow Stromal Cells from Ovariectomized Rats. J. Pharm. Pharmacol. 65, 1005-1013. doi:10.1111/ jphp. 12054

Tan, F. Z., and Dai, H. L. (2019). TAZ Accelerates Osteogenesis Differentiation of Mesenchymal Stem Cells via Targeting PI3K/Akt. Eur. Rev. Med. Pharmacol. Sci. 23, 81-88. doi:10.26355/eurrev_201908_18633

Tan, S., Xu, J., Lai, A., Cui, R., Bai, R., Li, S., et al. (2019). Curculigoside Exerts Significant Anti-arthritic E-ffects I-n vivo and I-n vitro via R-egulation of the JAK/STAT/NF-кB S-ignaling P-athway. Mol. Med. Rep. 19, 2057-2064. doi:10.3892/mmr.2019.9854

Teitelbaum, S. L. (2010). Stem Cells and Osteoporosis Therapy. Cell Stem Cell. 7, 553-554. doi:10.1016/j.stem.2010.10.004

Urbach, A., and Witte, O. W. (2019). Divide or Commit - Revisiting the Role of Cell Cycle Regulators in Adult Hippocampal Neurogenesis. Front. Cel Dev. Biol. 7, 55. doi:10.3389/fcell.2019.00055

Wang, Y., Zhao, L., Wang, Y., Xu, J., Nie, Y., Guo, Y., et al. (2012). Curculigoside Isolated from Curculigo Orchioides Prevents Hydrogen Peroxide-Induced Dysfunction and Oxidative Damage in Calvarial Osteoblasts. Acta Biochim. Biophys. Sin. 44, 431-441. doi:10.1093/abbs/gms014
Wang, N., Zhao, G., Zhang, Y., Wang, X., Zhao, L., Xu, P., et al. (2017). A Network Pharmacology Approach to Determine the Active Components and Potential Targets of Curculigo Orchioides in the Treatment of Osteoporosis. Med. Sci. Monit. 23, 5113-5122. doi:10.12659/msm.904264

Wang, N., Xue, P., Li, Z., and Li, Y. (2018). IRS-1 Increases TAZ Expression and Promotes Osteogenic Differentiation in Rat Bone Marrow Mesenchymal Stem Cells. Biol. Open 7, bio036194. doi:10.1242/bio.036194

Wang, N., Li, Y., Li, Z., Ma, J., Wu, X., Pan, R., et al. (2019). IRS-1 Targets TAZ to Inhibit Adipogenesis of Rat Bone Marrow Mesenchymal Stem Cells through PI3K-Akt and MEK-ERK Pathways. Eur. J. Pharmacol. 849, 11-21. doi:10.1016/j.ejphar.2019.01.064

Wang, N., Li, Y., Li, Z., Liu, C., and Xue, P. (2019). Sal B Targets TAZ to Facilitate Osteogenesis and Reduce Adipogenesis through MEK-ERK Pathway. J. Cel Mol Med. 23, 3683-3695. doi:10.1111/jcmm.14272

Wang, S., Liu, W., Wang, J., and Bai, X. (2020). Curculigoside Inhibits Ferroptosis in Ulcerative Colitis through the Induction of GPX4. Life Sci. 259, 118356. doi:10.1016/j.lfs.2020.118356

Wu, X.-Y., Li, J.-Z., Guo, J.-Z., and Hou, B.-Y. (2012). Ameliorative Effects of Curculigoside from Curculigo Orchioides Gaertn on Learning and Memory in Aged Rats. Molecules 17, 10108-10118. doi:10.3390/molecules 170910108

Wu, X., Li, S., Xue, P., and Li, Y. (2017). Liraglutide, a Glucagon-Like Peptide-1 Receptor Agonist, Facilitates Osteogenic Proliferation and Differentiation in MC3T3-E1 Cells through Phosphoinositide 3-kinase (PI3K)/protein Kinase B (AKT), Extracellular Signal-Related Kinase (ERK)1/2, and cAMP/protein Kinase A (PKA) Signaling Pathways Involving $\beta$-Catenin. Exp. Cel Res. 360, 281-291. doi:10.1016/j.yexcr.2017.09.018

Xu, Z., Sun, J., Tong, Q., Lin, Q., Qian, L., Park, Y., et al. (2016). The Role of ERK1/2 in the Development of Diabetic Cardiomyopathy. Int. J. Mol. Sci. 17, 2001. doi:10.3390/ijms17122001

Xue, P., Wu, X., Zhou, L., Ma, H., Wang, Y., Liu, Y., et al. (2013). IGF1 Promotes Osteogenic Differentiation of Mesenchymal Stem Cells Derived from Rat Bone Marrow by Increasing TAZ Expression. Biochem. Biophys. Res. Commun. 433, 226-231. doi:10.1016/j.bbrc.2013.02.088

Yu, B., Huo, L., Liu, Y., Deng, P., Szymanski, J., Li, J., et al. (2018). PGC-1a Controls Skeletal Stem Cell Fate and Bone-Fat Balance in Osteoporosis and Skeletal Aging by Inducing TAZ. Cell Stem Cell. 23 (2), 193-209.e5. doi:10.1016/ j.stem.2018.06.009

Yuan, T.-t., Xu, H.-t., Zhao, L., Lv, L., He, Y.-j., Zhang, N.-d., et al. (2015). Pharmacokinetic and Tissue Distribution Profile of Curculigoside after Oral and Intravenously Injection Administration in Rats by Liquid Chromatography-Mass Spectrometry. Fitoterapia 101, 64-72. doi:10.1016/ j.fitote.2014.12.012

Zanker, J., and Duque, G. (2019). Osteoporosis in Older Persons: Old and New Players. J. Am. Geriatr. Soc. 67, 831-840. doi:10.1111/jgs.15716

Zhang, Q., Zhao, L., Shen, Y., He, Y., Cheng, G., Yin, M., et al. (2019). Curculigoside Protects against Excess-Iron-Induced Bone Loss by Attenuating Akt-FoxO1dependent Oxidative Damage to Mice and Osteoblastic MC3T3-E1 Cells. Oxid Med. Cel Longev. 2019, 9281481. doi:10.1155/2019/9281481

Zheng, Y., and Pan, D. (2019). The Hippo Signaling Pathway in Development and Disease. Dev. Cel. 50, 264-282. doi:10.1016/j.devcel.2019.06.003

Conflict of Interest: The authors declare that the research was conducted in the absence of any commercial or financial relationships that could be construed as a potential conflict of interest.

Publisher's Note: All claims expressed in this article are solely those of the authors and do not necessarily represent those of their affiliated organizations, or those of the publisher, the editors, and the reviewers. Any product that may be evaluated in this article, or claim that may be made by its manufacturer, is not guaranteed or endorsed by the publisher.

Copyright (c) 2021 Wang, Li, Li, Li, Gao, Bao, Wang, Liu, Xue and Liu. This is an open-access article distributed under the terms of the Creative Commons Attribution License (CC BY). The use, distribution or reproduction in other forums is permitted, provided the original author(s) and the copyright owner(s) are credited and that the original publication in this journal is cited, in accordance with accepted academic practice. No use, distribution or reproduction is permitted which does not comply with these terms. 